\title{
Tracheoesophageal Puncture
}

National Cancer Institute

\section{Source}

National Cancer Institute. Tracheoesophageal Puncture. NCI Thesaurus. Code C15915.

A surgical procedure that creates a fistula between the trachea and esophagus in a patient who has previously undergone a total laryngectomy for the purpose of inserting a voice prosthesis to allow for esophageal speech. 